\title{
PRODUÇÃO DE UVAS SEM SEMENTES NO SEMIÁRIDO BRASILEIRO
}

Data de aceite: 01/09/2020

\section{Patrícia Coelho de Souza Leão}

Petrolina, PE cultivares trouxe novos desafios tecnológicos ao setor produtivo relacionados ao desenvolvimento de sistemas de produção que permitam a expressão do potencial máximo de produtividade e qualidade para cada cultivar.

PALAVRAS-CHAVE: Videira, Vitis sp., cultivares, viticultura tropical.

\section{SEEDLESS TABLE GRAPE PRODUCTION IN THE BRAZILIAN SEMI ARID}

ABSTRACT: Seedless table grape production in the Brazilian semiarid has advanced over the past two decades promoting the growth of grape consumption in the domestic market, and strengthening the grape's position among the main fruits exported by Brazil. Among the main technological challenges, we highlight the adaptation of seedless table grape cultivars to the semi-arid conditions of the São Francisco Valley, bringing together a set of superior agronomic characteristics such as high and stable yields in two crops per year, high fertility of buds, bunches and berries of adequate size, firm, crunchy texture, grapes with an appropriate balance between sugar and acid content and pleasant flavor. The offer of new seedless table grape cultivars is growing, increasing consumer interest in the diversity of berry shapes, colors and flavors. Grapes with special or exotic flavor classified as a gourmet type are offered in increasing volumes in the domestic and foreign markets. Currently, more than twenty of seedless table grapes cultivars are grown in the São Francisco Valley developed by Embrapa and private international genetic companies. The 
diversification of cultivars has brought new technological challenges for the productive sector related to the development of production systems that allow the expression of the maximum potential of productivity and quality for each cultivar.

KEYWORDS: Grapevine, Vitis sp., cultivars, tropical viticulture.

\section{1 | INTRODUÇÃO}

A viticultura destaca-se como uma das mais importantes cadeias produtivas da agricultura irrigada no Nordeste brasileiro, sendo que, em 2018, 31\% da produção nacional e 14\% da área cultivada estão concentrados no Submédio do Vale do São Francisco (IBGE 2020).

Ao longo da última década ocorreu substituição de cultivares de uvas de mesa, mas não houve ampliação significativa das áreas cultivadas. Segundo o IBGE (2020), os volumes produzidos na região passaram de 232,8 mil toneladas em 2004 para 469,8 mil toneladas em 2018, um incremento de mais de $50 \%$, enquanto a área cultivada, que em 2018 foi 10.068 ha, apresentou um crescimento neste mesmo período de 23\%. Aumento de volumes produzidos em menor área cultivada foi possível pela utilização de cultivares de uvas sem sementes produtivas e adaptadas para a produção de duas safras por ano que, associadas a outras tecnologias adotadas no sistema de produção, permitiram aumentar a produtividade e assegurar a qualidade da uva.

A uva é a terceira fruta na pauta de exportações do Brasil, atrás da manga e do melão e o Submédio do Vale do São Francisco responde por 99\% do total das exportações brasileiras dessa fruta desde o ano de 2002, com volumes que atingiram 45 mil toneladas em 2019 (COMEXTAT, 2020). Além da sua importância econômica, o cultivo da videira tem relevante papel social, pois gera até cinco empregos diretos por hectare. Em 2018, a uva e manga foram responsáveis pela criação de 100 mil empregos, segundo informações do Sindicato dos Produtores Rurais de Petrolina, sendo que a uva ocupou nos últimos anos o $1^{\circ}$ lugar na criação de novos postos de trabalho com 4.800, 4.410 e 4.540 empregos gerados nos anos de 2016, 2017 e 2018, respectivamente (BARBOSA, 2019).

Cada mercado apresenta preferências particulares que definem a decisão de compra de seus consumidores. Por exemplo, estudos publicados recentemente sobre as preferências do setor produtivo de uvas de mesa na China apontaram que a produtividade elevada, resistência a doenças, boa conservação pós-colheita, teor elevado de açúcares, bagas grandes ovaladas de cor vermelho intenso e negra, bem como sabor moscatel, são importantes atributos para adoção de novas cultivares de uvas de mesa, enquanto a ausência de sementes foi mencionada como uma característica de menor importância naquele país (WANG et al., 2017). Entretanto, esta é uma característica de grande importância na maioria dos países consumidores de uvas e um dos mais importantes objetivos no melhoramento genético de uvas de mesa (TÖPFER et al., 2011). 


\section{I CULTIVARES DE UVAS DE MESA SEM SEMENTES}

Um dos principais fatores limitantes ao crescimento das exportações pelo Submédio do Vale do São Francisco em meados da década de 1990 foi a produção concentrada em cultivares de uvas de mesa com sementes, especialmente na cultivar Italia. A necessidade de introdução, adaptação e viabilização técnica e econômica de cultivares de uvas sem sementes tornou-se, naquela época, o maior desafio para produtores, empresas e instituições de pesquisa. As pesquisas realizadas pela Embrapa Semiárido e instituições parceiras visando a introdução e avaliação de novas cultivares foi intensificada a partir de 1994.

As primeiras cultivares de uvas sem sementes comerciais 'Thompson Seedless', 'Sugraone' e 'Crimson Seedless' apresentaram características limitantes como baixa fertilidade de gemas, baixa produtividade, sensibilidade à rachadura de bagas e doenças, e produção concentrada em uma safra por ano com elevado grau de risco.

A redução significativa na rentabilidade econômica da produção de uvas dessas cultivares, consequência do alto custo de produção, perdas de safras causadas por chuvas e doenças, aliadas à cenários econômicos instáveis, trouxeram um segundo desafio para o setor produtivo e instituições de pesquisa: a necessidade de introduzir e desenvolver novas cultivares adaptadas para a produção de duas safras por ano e com produtividades elevadas e estáveis.

Deste modo, nesta última década, foram observadas grandes mudanças na cadeia produtiva de uvas de mesa, destacando-se a diversificação de cultivares e aumento da oferta de uvas sem sementes no mercado interno. Atualmente é cultivado um número superior a 20 cultivares de uvas de mesa desenvolvidas pela Embrapa e empresas privadas internacionais de melhoramento genético. As cultivares de uvas de mesa estrangeiras introduzidas na região têm um papel importante nas mudanças observadas no sistema de produção, com impacto positivo na rentabilidade econômica e fortalecimento da cadeia produtiva. Entretanto, a falta de adaptação de uma parte das seleções e cultivares às condições ambientais do semiárido tropical, a suscetibilidade à doenças, o custo da licença (royalties) para a sua produção e as restrições quanto ao tamanho das áreas cultivadas e número de empresas licenciadas, impostas pelas empresas privadas de melhoramento, enfatizam necessidade de independência tecnológica do país por meio do desenvolvimento de cultivares de uvas de mesa brasileiras e adaptadas às condições ambientais das principais regiões produtoras.

Vale a pena destacar a importância das seguintes cultivares desenvolvidas por diferentes empresas internacionais de melhoramento genético, encontradas no Submédio do Vale do São Francisco e nos principais países produtores de uvas de mesa:

- Uvas brancas: Arra $15^{\circledast}\left(\right.$ Grapa), Sugar Crisp ${ }^{\circledast}$, Sweet Globe ${ }^{\circledast}$, Cotton Candy ${ }^{\circledast}$ (International Fruit Genetics - IFG) e Autumm Crisp ${ }^{\circledast}$ (Sun Word) 
- Uvas vermelhas: Sweet Celebration ${ }^{\circledR}$, Candy Snaps ${ }^{\circledR}$ (IFG), Timco ${ }^{\circledR}$ (Sheegene), Scarlotta Seedless ${ }^{\circledR}$ (Sun World)

- Uvas negras: Sweet Saphire ${ }^{\circledR}$ (IFG), Sable ${ }^{\circledR}$, Midnight Beauty ${ }^{\circledR}$ (Sun World).

Estas são citadas como exemplos, uma vez que mais de 70 cultivares desenvolvidas por estas empresas estão disponíveis para produtores licenciados.
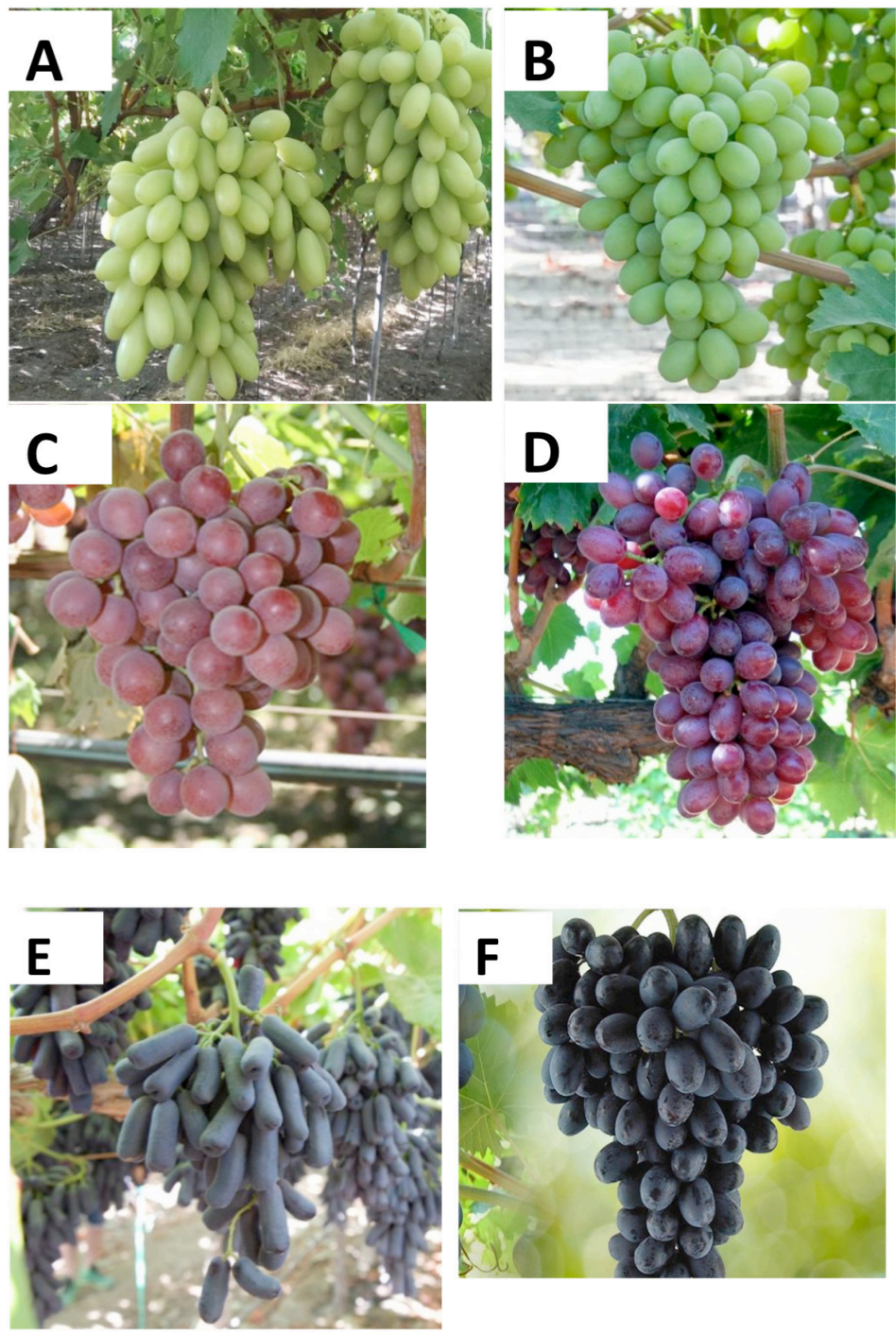

Figura 1. Cachos de uvas de cultivares de programas privados internacionais de melhoramento genético: Arra $15^{\circledR}(A)$, Sugar Crisp ${ }^{\circledR}(B)$, Sweet Celebration ${ }^{\circledast}(C)$, Scarlotta Seedless ${ }^{\circledR}(D)$, Sweet Saphire ${ }^{\circledR}(E)$ e Midnight Beauty Seedless ${ }^{\circledR}(F)$. Fotos: Patrícia Coelho de Souza Leão (A), IFG (B, C e E) e Sun World (D e F) 
A seguir é apresentada uma breve descrição das cultivares de uvas de mesa desenvolvidas pela Embrapa, às quais são cultivares protegidas e públicas, ou seja: estão disponíveis para todos os produtores que podem adquirir as mudas em viveiros credenciados pela Embrapa.

\section{BRS Vitória}

A cultivar BRS Vitória foi desenvolvida pelo programa de melhoramento genético da Embrapa e recomendada para cultivo em 2012 (MAIA et al., 2012), adaptando-se às condições tropicais semiáridas do Submédio do Vale do São Francisco. As videiras apresentam alta fertilidade de gemas, produtividade médias de 50 a 60 ton/ha/ano, moderada tolerância ao míldio (Plasmopora viticola), cachos e bagas de tamanho médio (Figura 2), teor de sólidos solúveis acima de $19^{\circ}$ Brix e acidez titulável entre 0,6 a 0,8 g de ácido tartárico/100mL (LEÃO; LIMA, 2016).

A área cultivada no Submédio do Vale do São Francisco avançou rapidamente nos últimos cinco anos, estimando-se atualmente área superior a 2.000ha, destacandose como a mais importante cultivar de uva de mesa desenvolvida pela Embrapa e ainda uma das mais importantes uvas sem sementes cultivadas no Brasil. A cultivar BRS Vitória tem despertado interesse dos consumidores nos mercados interno e externo pelo sabor especial e agradável, sendo muito apreciada em mercados especializados de uvas tipo gourmet.

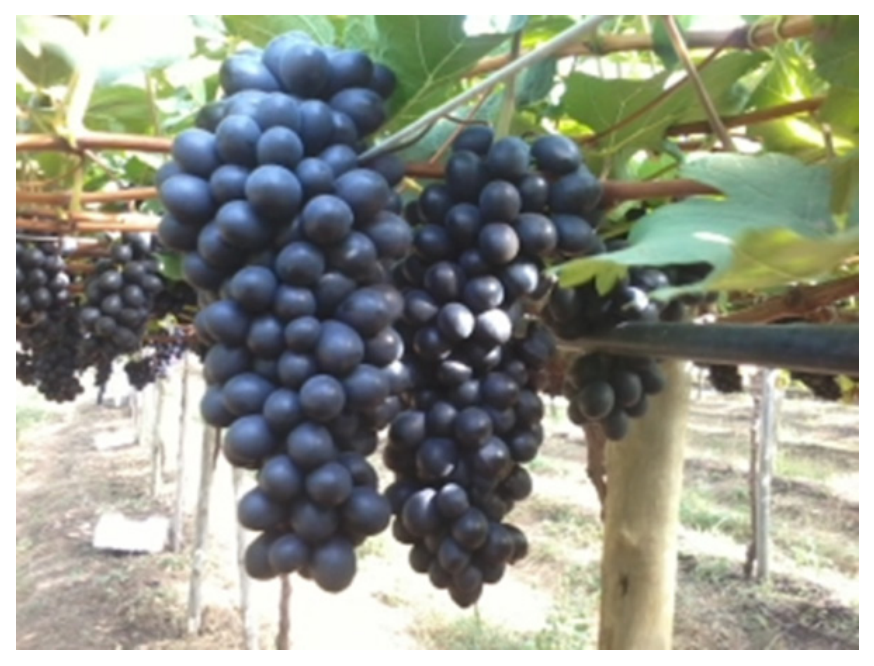

Figura 2. Cacho de uva 'BRS Vitória'. Foto: Patrícia Coelho de Souza Leão 


\section{BRS Ísis}

A cultivar BRS Isis foi lançada em 2013 (RITSCHEL et al., 2013) e caracteriza-se como uma uva de mesa de cor vermelha e traços de sementes grandes (Figura 3). No Vale do Submédio São Francisco, a duração média do ciclo de produção foi de 127 dias da poda até a colheita, podendo ser classificada como uma cultivar de ciclo tardio.

As videiras são vigorosas, apresentam índices de fertilidade de gemas elevados

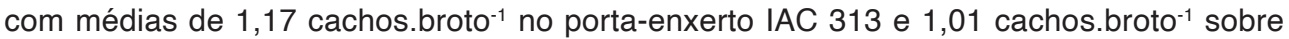
'SO4', produtividades em torno de 60 ton/ha/ano (LEÃO et al. 2016).

Os cachos têm tamanho mediano e formato cilíndrico-alado ou cilíndrico com massa em torno de $400 \mathrm{~g}$. As bagas tem forma elíptica larga com dimensões em torno de $28 \mathrm{~mm}$ $X 20 \mathrm{~mm}$ que podem variar de acordo com o porta enxerto e manejo do vinhedo. As bagas apresentam película grossa, polpa firme e incolor e sabor neutro.

As uvas devem ser colhidas com teor de sólidos solúveis (SS) a partir de $16^{\circ}$ Brix e acidez titulável (AT) entre 0,4 à $0,6 \mathrm{~g} \cdot 100 \mathrm{~mL}^{-1}$. Estes valores correspondem à relação SS/AT acima de 25, evidenciando sabor agradável (LEÃO et al., 2016). Por outro lado, algumas características indesejáveis são o desenvolvimento de sementes e a sensibilidade a rachadura de bagas quando ocorrem chuvas na fase final de maturação da uva.

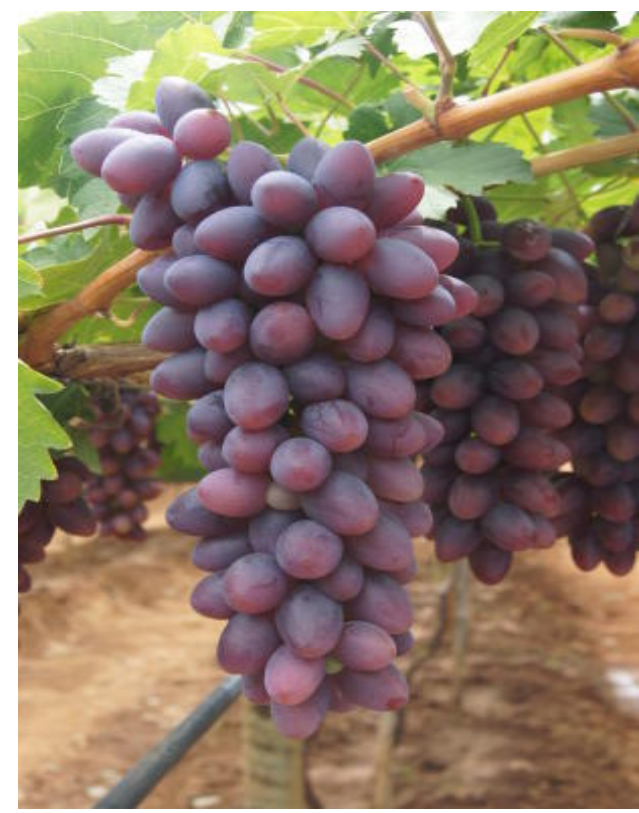

Figura 3. Cacho de uva 'BRS Ísis'. Foto: Embrapa 


\section{BRS Tainá}

A cultivar BRS Tainá será lançada e recomendada para cultivo na região do Submédio do Vale do São Francisco no segundo semestre de 2020. Esta é a primeira cultivar de uva de mesa desenvolvida pela Embrapa realizando-se todas as etapas do melhoramento genético em condições semiáridas. Portanto, é considerada a primeira cultivar brasileira "sertaneja".

As videiras são vigorosas, apresentam fertilidade intermediária de gemas em torno

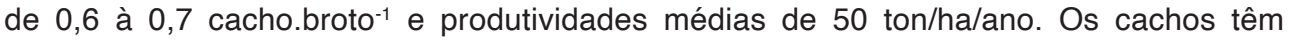
tamanho médio, formato cônico, medianamente compacto e pedúnculo curto (Figura 4). Por sua vez, as bagas tem tamanho médio $(26 \mathrm{~mm}$ X 20mm), forma elipsóide-larga, cor verde amarelada, aderência moderada ao pedicelo, película fina, textura da polpa firme e crocante, sabor neutro e sementes rudimentares minúsculas (LEÃO et al. 2020).

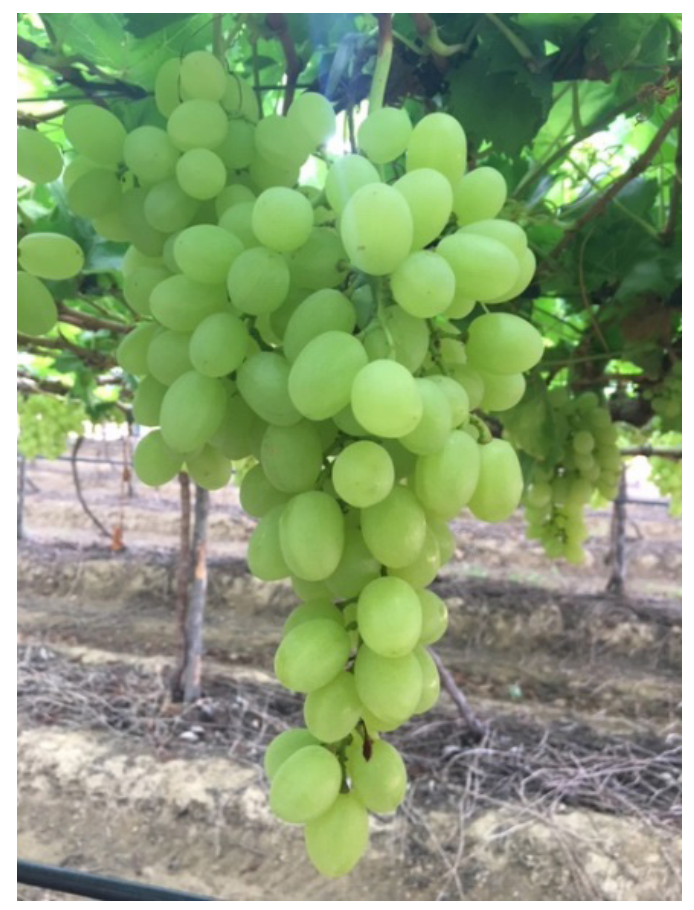

Figura 4. Cacho de uva ‘BRS Tainá'. Foto: Patrícia Coelho de Souza Leão

O principal problema apresentado pela cultivar BRS Tainá foi a suscetibilidade à rachadura, que pode ocorrer a partir da base da baga, no ponto de inserção da caliptra ocorrendo em condições de excesso de água no solo, sendo necessário ajustar o manejo nutricional e de irrigação, determinando e controlando níveis adequados de umidade no solo. 


\section{BRS Clara}

Cultivar de uva sem sementes desenvolvida pela Embrapa Uva e Vinho e lançada em 2003 (CAMARGO et al., 2003).

No Submédio do Vale do São Francisco, o ciclo fenológico desde a poda até a colheita foi aproximadamente de 100 dias o que caracteriza 'BRS Clara' como cultivar precoce.

As videiras são vigorosas, apresentam elevada fertilidade de gemas e produtividade entre 40 à 50 ton/ha em duas safras ao ano. Os cachos apresentam tamanho médio com massa em torno de $350 \mathrm{~g}$, formato cônico, às vezes alado e pedúnculo longo. As bagas são pequenas $(21 \mathrm{~mm} \times 15 \mathrm{~mm})$, elípticas, verde amareladas, tem película de espessura média, polpa incolor, textura firme e crocante, sabor neutro e adstringente. As sementes rudimentares são pequenas mas lignificadas e de cor marrom, portanto são perceptíveis ao cortar a baga. Apresentam tolerância a rachadura da baga quando a maturação e colheita ocorre em períodos de chuva.

As uvas da cultivar BRS Clara apresentam elevado potencial glucométrico, com teor de sólidos solúveis que variou entre 17 a $21^{\circ}$ Brix e a acidez titulável de 0,43 a 0,86 g.100 $\mathrm{mL}^{-1}$ em cinco ciclos de produção (LEÃO 2018). Recomenda-se que a colheita seja realizada com teor de sólidos solúveis entre 18 e $19^{\circ}$ Brix e acidez titulável inferior a 0,8 g.100 $\mathrm{mL}^{-1}$ para reduzir a forte adstringência das bagas que é uma das características negativas desta cultivar.

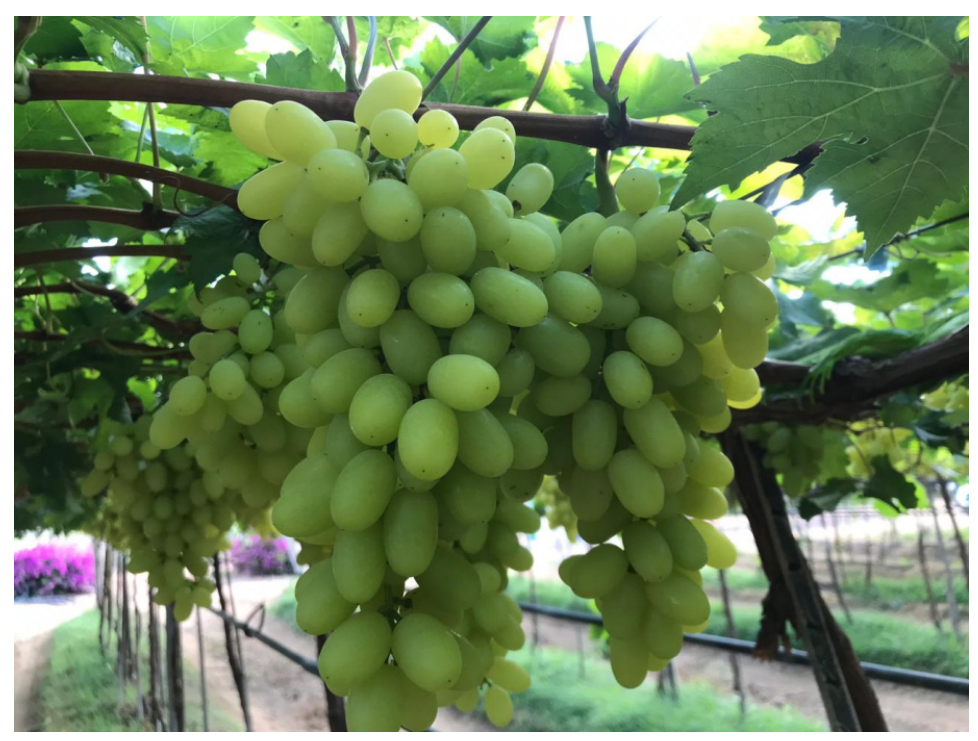

Figura 5. Cachos de uva 'BRS Clara'. Foto: Patrícia Coelho de Souza Leão 


\section{BRS Melodia}

A cultivar 'BRS Melodia' foi recomendada pela Embrapa Uva e Vinho para cultivo na região sul do Brasil sob cobertura plástica (MAIA et al. 2019) e encontra-se em fase de validação no Submédio do Vale do São Francisco.

Constitui uma alternativa de uva de mesa sem sementes que atende a mercados de uvas do tipo gourmet pelo sabor especial de mix de frutas vermelhas.

A avaliação de seu comportamento agronômico e qualidade da uva na região do Submédio do Vale do São Francisco encontra-se em fase inicial, portanto ainda não existem informações consolidadas pela pesquisa nesta região.

Entretanto as observações preliminares em campo demonstram o elevado potencial que esta cultivar apresenta pela sua elevada fertilidade de gemas, cachos e bagas de tamanho médio, cor rosada, e sabor especial e muito atrativo para o consumo in natura.

A sua recomendação para cultivo em condições semiáridas está prevista para 2021, quando os produtores poderão adquirir mudas nos viveiristas credenciados pela Embrapa.

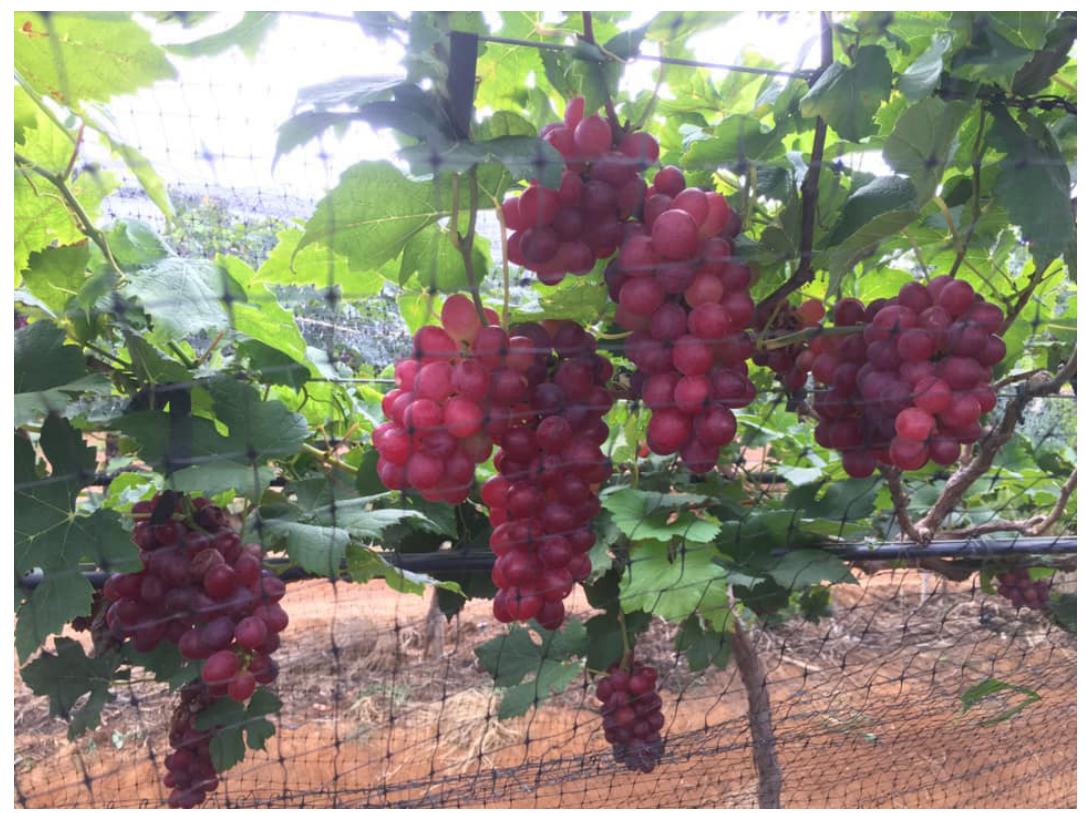

Figura 5. Cachos de uva ‘BRS Melodia'. Foto: Newton Matsumoto

\section{3| DESAFIOS E PERSPECTIVAS}

A diversificação de cultivares e avanços na produção de uvas sem sementes observados na última década resultaram em impactos positivos no fortalecimento da cadeia produtiva. A oferta de novas cultivares consolidou o consumo de uvas sem sementes no 
Brasil e reduziu os volumes importados, especialmente do Chile. Segundo informações da Seção de Economia e Desenvolvimento da CEAGESP (Companhia de Entrepostos e Armazéns Gerais de São Paulo), principal entreposto de comercialização da fruta no país, de janeiro a junho de 2019, foram comercializadas 6,5 mil toneladas de uvas sem sementes brasileiras enquanto as importadas foram apenas 1,1 mil toneladas.

No mercado externo nota-se a tendência, a partir de 2019, de oferta regular de diferentes cultivares de uvas de mesa ao longo do ano, incluindo os meses do primeiro semestre em que a oferta é restrita nos mercados importadores (LIMA et al., 2019). Além disso, existe o interesse crescente pela diversidade, ou seja, uvas com diferentes formas, cores e principalmente sabores especiais e exóticos. Estas uvas tipo gourmet e destinadas a nichos de mercado apresentam forte tendência de crescimento. Entretanto, cultivares que apresentam cor branca, bagas grandes, firmes, crocantes, sabor neutro e agradável continuam sendo àquelas que encontram maior espaço no mercado externo. Do ponto de vista do produtor, a fertilidade de gemas e produtividades elevadas e estáveis são características imprescindíveis, mas a tolerância ou resistência as principais doenças que afetam a videira, ainda são objetivos a serem alcançados nas futuras cultivares de uvas de mesa, uma vez que as cultivares atuais não apresentam resistência a doenças.

A oferta de novas cultivares de uvas brasileiras e internacionais aumenta a competição entre cultivares, promovendo a rápida substituição daquelas com características indesejáveis e menor aceitação entre os consumidores, por outras com características superiores capazes de despertar interesse dos consumidores e alcançar preços mais elevados. Portanto, a viticultura tropical no semiárido apresenta um movimento dinâmico crescente que exige investimentos e esforços públicos e privados no desenvolvimento de sistemas de produção adaptados para as características genéticas e necessidades de cada cultivar.

Aspectos do sistema de produção como sistema de condução, cultivo protegido, espaçamento, porta-enxerto, tipos de poda, densidade de brotos e de cachos, uso de reguladores de crescimento, manejo de água e nutrientes, controle fitossanitário, determinação do ponto de colheita, e uso de tecnologias para aumentar a vida de prateleira precisam ser ajustados para cada uma das cultivares de uvas de mesa.

Por outro lado, Lima et al (2019) mencionam as demandas apontadas no Plano Nacional de Desenvolvimento da Fruticultura os quais são também os principais gargalos tecnológicos da viticultura tropical: promoção e certificação de mudas; desenvolvimento e uso de insumos e agentes de controle biológico/naturais; agregação de valor às frutas e seus derivados; redução de perdas em pós-colheita; desenvolvimento de novas embalagens e formas de comercialização atraentes ao consumidor; diversificação varietal; estruturação de observatórios, cadastros e bases de dados para tomada de decisão pelos atores das cadeias produtivas; ampliação do uso de ferramentas da fruticultura de precisão; mecanização na fruticultura e uso de equipamentos de apoio para o monitoramento, com 
base na adoção de tecnologias da informação e do conhecimento; fortalecimento do uso intensivo de sistemas de alerta e tecnologias de mitigação de danos pela redução do impacto de riscos climáticos e perdas por fatores bióticos e abióticos associados ao clima; e desenvolvimento e promoção de indicações geográficas de frutas.

Os desafios tecnológicos apresentados ressaltam a importância da organização dos diferentes elos da cadeia produtiva, das associações e cooperativas de pequenos e médios produtores e o fortalecimento de parcerias entre instituições públicas e privadas para garantir o crescimento sustentável da produção de uvas de mesa no semiárido brasileiro.

\section{REFERÊNCIAS}

CAMARGO, U. A.; NACHTIGAL, J. C.; MAIA, J. D. G.; OLIVEIRA, P .R. D. de; PROTAS, J. F. da S. BRS Clara: nova cultivar de uva de mesa branca sem semente. Bento Gonçalves:Embrapa Uva e Vinho, 2003. 4 p. (Embrapa Uva e Vinho. Comunicado Técnico, 46).

COMEXSTAT. Sistema de Estatísticas do Comércio Exterior. Disponível em: <http://comexstat.mdic. gov.br/pt/geral>. Acesso em: agosto de 2020.

BARBOSA, E. Fruticultura alavanca empregos no Vale do São Francisco. Folha de Pernambuco. https://www.folhape.com.br/economia/fruticultura-alavanca-empregos-no-vale-do-saofrancisco/123315/. Acesso em 22.06.2020.

IBGE (INSTITUTO BRASILEIRO DE GEOGRAFIA E ESTATÍSTICA). Pesquisa Produção Agrícola Municipal. In: Sidra: sistema IBGE de Recuperação Automática. Rio de Janeiro, 2020. Disponível em: <https://sidra.ibge.gov.br/pesquisa/pam/tabelas>. Acesso em: agosto de 2020.

LEÃO, P. C. de S.; BORGES, R. M. E.; MELO, N. F. de; BARBOSA, M. A. G.; LIMA, M. A. C. de; FLORES, R. C.; MARQUES, A. T. B. 'BRS Tainá': nova cultivar de uvas sem sementes de cor branca para o Vale do São Francisco. Petrolina:Embrapa Semiárido, 2020.

LEAO, P. C. de S.; NUNES, B. T. G.; SOUZA, E. M. C. de; REGO, J. I. de S.; NASCIMENTO, J. H. B. do. BRS Isis: new seedless grape cultivar for the tropical viticulture in Northeastern of Brazil. BIO Web of Conferences, v. 7, p. 1-4, oct. 2016. Edição do 39 World Congress of Vine and Wine, Bento Gonçalves, oct. 2016.

LEÃO, P. C. de S.; LIMA, M. A. C. de. Uva de mesa sem sementes 'BRS Vitória': comportamento agronômico e qualidade dos frutos no Submédio do Vale do São Francisco. Embrapa Semiárido:Petrolina. Comunicado Técnico, 168. 5p. 2016.

LEAO, P. C. de S. Uva: adaptada ao cultivo Cultivar HF, v. 15, n. 108, p. 26-29, fav./mar. 2018.

LIMA, M. A. C. de; GUERRA, C. C.; BIANCHINI, F.; LEAO, P. C. de S. Pesquisa, desenvolvimento e inovação para a produção tropical de uvas para mesa, vinho e suco: situação atual e oportunidades. Petrolina: Embrapa Semiárido, 2019. 62 p. il. (Embrapa Semiárido. Documentos, 293).

MAIA, J. D. G.; RITSCHEL, P. S.; CAMARGO, U. A.; SOUZA, R. T. de; GROHS, D. S.; FAJARDO, T. V. M. BRS Melodia: nova cultivar de uvas sem sementes, com sabor especial de mix de frutas vermelhas, recomendada para cultivo na Serra Gaúcha, em cobertura plástica.Bento Gonçalves:Embrapa Uva e vinho, 22 p. il., color., 2019. (Embrapa Uva e Vinho, Circular Técnica, 144). 
MAIA, J.D.G.; RITSCHEL, P.; CAMARGO, U.A.; SOUZA, R.T. de S.; FAJARDO, T.V.M.; NAVES, R. de L.; GIRARDI, C.L. 'BRS Vitória' Nova cultivar de uva de mesa sem sementes com sabor especial e tolerante ao míldio. Bento Gonçalves: Embrapa Uva e Vinho, 2012, 12 p. il., color. (Embrapa Uva e Vinho, Comunicado Técnico, 126).

RITSCHEL, P.S.; MAIA, J.D.G.; CAMARGO, U.A.; SOUZA, R.T. de; FAJARDO, T.V. M.; NAVES, R. de L.; GIRARDI, C.L. BRS Isis: nova cultivar de uva de mesa vermelha, sem sementes e tolerante ao míldio. Bento Gonçalves: Embrapa Uva e Vinho, 2013. 20 p. il., color. (Embrapa Uva e Vinho. Comunicado Técnico, 143).

TÖPFER, R.; HAUSMANN, L.; HARST, M.; MAUL, R.; ZYPRIAN, E.; EIBACH, R. 2011. New Horizons for Grapevine Breeding. Fruit, Vegetable and Cereal Science and Biotechnology, 5:1, 79-100.

WANG, Z.; ZHOU, J.; XU, X.; PERL, A.; CHEN, S.; MA, H. 2017. Adoption of table grape cultivars: An attribute preference study on Chinese grape growers. Scientia Horticulturae 216, 66-75. 